\title{
Glycine receptors control the generation of projection neurons in the developing cerebral cortex
}

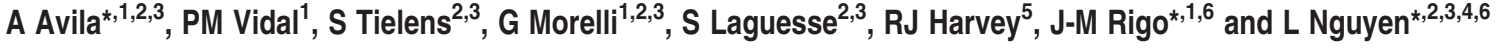

The development of the cerebral cortex requires coordinated regulation of proliferation, specification, migration and differentiation of cortical progenitors into functionally integrated neurons. The completion of the neurogenic program requires a dynamic interplay between cell intrinsic regulators and extrinsic cues, such as growth factor and neurotransmitters. We previously demonstrated a role for extrasynaptic glycine receptors (GlyRs) containing the $\alpha 2$ subunit in cerebral cortical neurogenesis, revealing that endogenous GlyR activation promotes interneuron migration in the developing cortical wall. The proliferative compartment of the cortex comprises apical progenitors that give birth to neurons directly or indirectly through the generation of basal progenitors, which serve as amplification step to generate the bulk of cortical neurons. The present work shows that genetic inactivation of Glra2, the gene coding the $\alpha 2$ subunit of GlyRs, disrupts dorsal cortical progenitor homeostasis with an impaired capability of apical progenitors to generate basal progenitors. This defect results in an overall reduction of projection neurons that settle in upper or deep layers of the cerebral cortex. Overall, the depletion of cortical neurons observed in Glra2-knockout embryos leads to moderate microcephaly in newborn Glra2-knockout mice. Taken together, our findings support a contribution of GlyR $\alpha 2$ to early processes in cerebral cortical neurogenesis that are required later for the proper development of cortical circuits.

Cell Death and Differentiation (2014) 21, 1696-1708; doi:10.1038/cdd.2014.75; published online 13 June 2014

The cerebral cortex develops from the forebrain and contains different classes of neurons distributed within layers that are regionally organized into sensory, motor and association areas. Cerebral cortex layering arises inside-out as progenitors give birth to successive waves of pyramidal projection neurons in the dorsal telencephalon ${ }^{1}$ and GABAergic interneurons in the ventral forebrain. ${ }^{2}$ Projection neurons migrate radially to settle in appropriate layers of the cortical plate (CP) from where they grow axonal projections towards cortical or subcortical targets. Interneurons migrate from the ganglionic eminences along multiple tangential paths to integrate local cortical networks. More generally, the development of the cortex progresses through successive steps including proliferation, specification, migration and neuronal differentiation. Disrupting the completion of one or several of these cellular events may lead to severe cortical malformations underlying neurological disorders characterized by learning and intellectual disability or epilepsy. ${ }^{1,3}$

The generation of cortical neurons requires the integration of genetic programs with multiple signals present in the extracellular environment of cortical progenitors. ${ }^{4}$ Neurotransmitters belong to their biochemical environment during early stages of corticogenesis. ${ }^{5}$ These molecules control neurotransmission in the mature central nervous system (CNS), whereas they contribute to various developmental processes before synaptogenesis in the developing nervous system. ${ }^{6-8}$ Accumulating work has provided evidences that GABA and glutamate control neural progenitor proliferation by activating ligand-gated ion channel (LGIC) receptors. ${ }^{9-11}$ Recent findings have further highlighted the general molecular mechanisms triggered by neurotransmitters to modulate cell proliferation, cell migration ${ }^{12,13}$ and more globally changes in cell morphology. ${ }^{14}$ It is noteworthy that GABA, the main inhibitory neurotransmitter in the adult brain, depolarizes and excites immature neurons during embryonic development due to developmental regulation of the transmembrane $\mathrm{Cl}^{-}$gradient. ${ }^{15,16} \mathrm{GABA}_{\mathrm{A}}$ and $\mathrm{GABA} \mathrm{C}_{\mathrm{C}}$ receptors $\left(G A B A_{A} R s\right.$ and $G A B A_{C} R s$, respectively) are expressed by immature cortical cells, ${ }^{17}$ and their activation leads to membrane depolarization that triggers activation of voltage-gated $\mathrm{Ca}^{2+}$ channels. This is further responsible for local intracellular $\mathrm{Ca}^{2+}$ elevations, which in turn modulates the rate of cell proliferation. ${ }^{10,11}$ Strikingly, Gad1-knockout mice, lacking glutamate decarboxylase (GAD) 67 (the enzyme that produces GABA), do not have major cortical malformations. This suggests existence of compensatory mechanisms, such as activation of other neurotransmitter receptors. Within this

\footnotetext{
${ }^{1}$ BIOMED Research Institute, Hasselt University, Diepenbeek, Belgium; ${ }^{2}$ GIGA-Neurosciences, University of Liège, C.H.U. Sart Tilman, Liège, Belgium; ${ }^{3}$ Interdisciplinary Cluster for Applied Genoproteomics (GIGA-R), University of Liège, C.H.U. Sart Tilman, Liège, Belgium; ${ }^{4}$ Walloon Excellence in Lifesciences and Biotechnology (WELBIO), University of Liège, C.H.U. Sart Tilman, Liège, Belgium and ${ }^{5}$ Department of Pharmacology, UCL School of Pharmacy, 29-39 Brunswick Square, London, UK

*Corresponding author: L Nguyen or J-M Rigo or A Avila, Developmental Neurobiology GIGA-Neurosciences, Avenue de l'Hôpital, 1 bâtiment B36 + 1, Liege 4000, Belgium. Tel: + 324 3665987; Fax: + 324 3665912; E-mail: Inguyen@ulg.ac (LN) or Tel: + 32112680 04; Fax: + 32112681 99; E-mail: jeanmichel.rigo @uhasselt.be (J-MR) or Tel: + 1647740 0368; E-mail: arielsalvatore@gmail.com (AA)

${ }^{6}$ These senior authors contributed equally to the work.

Abbreviation: APs, apical progenitors; BPs, basal progenitors; BrdU, bromodeoxyuridine; CNS, central nervous system; CP, cortical plate; E15, embryonic day 15; GABAARs, GABA type A receptors; GABACRs, GABA type C receptors; GAD, glutamate decarboxylase; GIGA-R, Interdisciplinary cluster for applied genoproteomics; Glra2, glycine receptor alpha 2 gene; GlyRs, glycine receptors; IZ, intermediate zone; KO, knockout; LGIC, ligand-gated ion channel; mM, millimolar; NDS, normal donkey serum; NIH, National Institute of Health; P0, postnatal day zero; SVZ, subventricular zone; VGCCs, voltage-gated $\mathrm{Ca}^{2+}$ channels; WT, wild type Received 21.2.14; revised 10.4.14; accepted 29.4.14; Edited by P Salomoni; published online 13.6.14
} 
context, GlyRs are strong candidates. They are composed of pentameric assembly of alpha ( $\alpha 1-\alpha 4)$ and/or beta subunits $(\beta)$ forming an ionic pore that selectively controls, such as $\mathrm{GABA}_{\mathrm{A}} \mathrm{Rs}$, trans-membrane fluxes of chloride and bicarbonate ions. ${ }^{18}$ In the nervous system, GlyRs are not only gated by glycine but also taurine, D-serine or $\beta$-alanine, which are abundant in the developing brain (reviewed in Avila et al. ${ }^{19}$ ). Postsynaptic GlyRs control synaptic transmission of sensory stimuli and are involved in the generation of motor outputs in the spinal cord. ${ }^{20}$ These receptors are also expressed in the hippocampus ${ }^{21-25}$ and in the cortex. ${ }^{26,27}$ Importantly, GlyR heteromers cluster at the presynaptic membrane to control neurotransmitter release in the medial nucleus of trapezoid body in the brainstem, ${ }^{28}$ the spinal cord ${ }^{29,30}$ and the ventral tegmental area. ${ }^{31}$ During neurogenesis, extrasynaptic GlyRs and their ligands are present in the forebrain ${ }^{32,33}$ as well as in neurogenic regions of the postnatal brain. ${ }^{34-37}$ However, we have limited information about their physiological role in this specific context (reviewed in Avila et al. ${ }^{19}$ ). Studies conducted in the developing CNS suggest that most GlyRs are homomers, containing the $\alpha 2$ subunit. This was further confirmed in cortical interneurons, where the activation of GlyRs contributes to tangential migration. ${ }^{32}$ A similar role for GlyRs has been suggested for cortical projection neurons, ${ }^{38}$ but the contribution of these receptors to proliferation and specification of cortical progenitors has not yet been determined. Cortical projection neurons are generated by different subpopulations of progenitors that are located around lateral ventricles. ${ }^{39}$ The majority of $\mathrm{VZ}$ progenitors are apical progenitors (APs, also named radial glia progenitors) and they express the transcription factors Pax6 and Sox2. ${ }^{40,41}$ Early stages of corticogenesis are characterized by a rapid expansion phase, during which APs undergo symmetric cell division to selfrenew. As the cortical wall expands, they move into a neurogenic phase during which most of them divide asymmetrically to simultaneously self-renew and generate either a neuron (direct neurogenesis) or a Tbr2-expressing basal progenitors (BP, also named intermediate progenitor) that further divides in the SVZ. These cells act as transit-amplifying progenitors and produce the majority of projection neurons that populate all cortical layers ${ }^{42}$ via indirect neurogenesis. ${ }^{43,44}$ To date, the molecular mechanisms that control the proliferation and fate choice of APs and BPs, and thus the generation of projection neurons, remain poorly understood.

The present work explores the role of GlyRs in the generation of excitatory projection neurons. We demonstrate that cortical progenitors (APs and BPs) express functional GlyRs containing the $\alpha 2$ subunit and that a genetic disruption of the corresponding gene (Glra2) reduces the ability of APs to generate BPs and favors differentiation of BPs into neurons. These defects contributed to an overall reduction of projection neuron output in the developing cortex, leading to moderate microcephaly in newborn Glra2-knockout mice.

\section{Results}

Functional glycine receptors are expressed by dorsal cortical progenitors. Glycine receptors composed of $\alpha 2$ subunits are widely distributed in the forebrain. ${ }^{33}$ In particular, cortical plate neurons and Cajal-Retzius cells express functional $\alpha 2 \beta$ heteromeric GlyRs ${ }^{37}$ that when activated results in membrane depolarization. ${ }^{34,35}$ We recently demonstrated that endogenous glycine activates $\alpha 2$-containing GlyRs to promote cortical interneuron migration. ${ }^{32}$ In this study, we investigated the distribution of GlyRs in dorsal cortical progenitors and their progeny by using confocal microscopy. Immunolabeling with antibodies against $\alpha 2$ subunits revealed a widespread distribution of GlyRs in the embryonic cortex at E13 with stronger staining in some VZ cells (Figure 1a, left side). We did not detect $\alpha 2$ subunit immunostaining in the cortical anlage of age-matched Glra2-knockout embryos (Glra2 KO) (Figure 1a, right side). Similar labeling was obtained with pan-GlyR alpha antibodies ${ }^{45}$ (e.g., mAb4a clone; data not shown). This result suggests that dorsal progenitors and projection neurons express GlyRs enriched in $\alpha 2$ subunits, as previously shown for cortical interneurons. ${ }^{32}$ Immunohistochemical analyses performed at later development stages showed a progressive restriction of GlyRs expression to peri-ventricular region and $\mathrm{CP}$ (E15), then mostly in $\mathrm{CP}$ (E17) and further layer $V$ neurons (P0; Figure 1b). We performed in utero electroporation of E13 WT embryos with plasmids driving GFP under a regulatory sequence of $B L B P$, a gene expressed by APs. Immunolabelings were performed on the brain collected the following day to allow identification of APs (that co-express Pax6, data not shown) and their direct Tbr2-positive BP progeny, thanks to GFP expression. Single confocal planes showed dotted expression of GlyR $\alpha 2$ in ventricle-lining APs (Tbr2-negatives and GFP-positives) as well as in BPs located in the SVZ (Tbr2-, and GFP-positives) (Figure 1c). Whole-cell patch clamp recordings were performed to assess expression of functional $\alpha 2$-containing GlyRs in these cortical progenitors. For this purpose, brain slice cultures from E13 embryos were infected with GFPexpressing retroviruses that integrate into all cycling progenitors in VZ (APs) and SVZ (BPs) (Figure 1d). Under these conditions, application of glycine triggered fast-activating currents that had a slow desensitization in the presence of the ligand in both VZ and SVZ cells (Figure 1e). We performed additional recordings and showed that the maximal average generated current in BPs was $324 \pm 140$ pA. The concentration-response curve was characterized by an $E_{50}$ of $72 \pm 10 \mu \mathrm{M}$ and a Hill coefficient of $1.7 \pm 0.2$ (Figure 1f). Moreover, glycine-elicited currents were blocked by bath application of strychnine at $1 \mu \mathrm{M}$ (Figure 1g), supporting the specific activation of GlyRs. During development, most neuronal progenitors are characterized by an inverted chloride-gradient, and activation of GlyRs or $\mathrm{GABA}_{A} \mathrm{Rs}$ triggers membrane depolarization and local $\mathrm{Ca}^{2+}$ influxes through opening of voltage-gated $\mathrm{Ca}^{2+}$ channels (VGCCs). ${ }^{32,46}$ We therefore tested whether GlyRs activation promotes membrane depolarization and modulates intracellular $\mathrm{Ca}^{2+}$ concentrations in dorsal cortical progenitors (APs and BPs). Brain slices were loaded with Fluo4 AM, and time-lapse recordings were made to assess intracellular $\mathrm{Ca}^{2+}$ oscillations upon application of glycine. Glycine $(300 \mu \mathrm{M})$ induced short and reversible rises in intracellular $\mathrm{Ca}^{2+}$ in cells distributed throughout the cortical wall. Prominent signals were recorded in sparse cells located in the VZ and SVZ (Figure 1h), among which some were APs and BPs, respectively. Similar results were obtained after bath application of GABA $(100 \mu \mathrm{M}$, data not shown) as previously reported. ${ }^{10}$ 
Genetic inactivation of GIra2 induces moderate microcephaly in newborn mice. Gross morphological analyses revealed that newborn Glra2-KO mice had reduced brain size (Figures 2a-d) with significant thinning of the cerebral cortical wall (Figure 2e) and shrinking of the striatal area (Figure 2f). We previously demonstrated that cortical

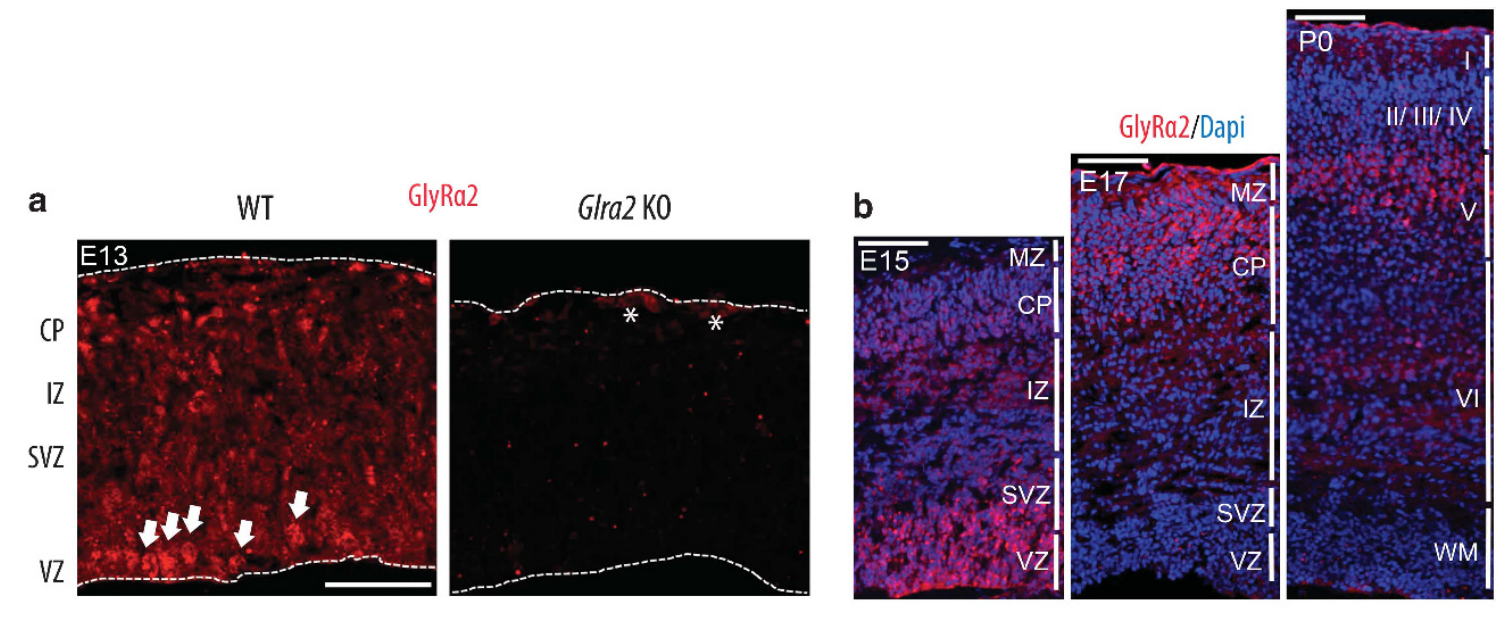

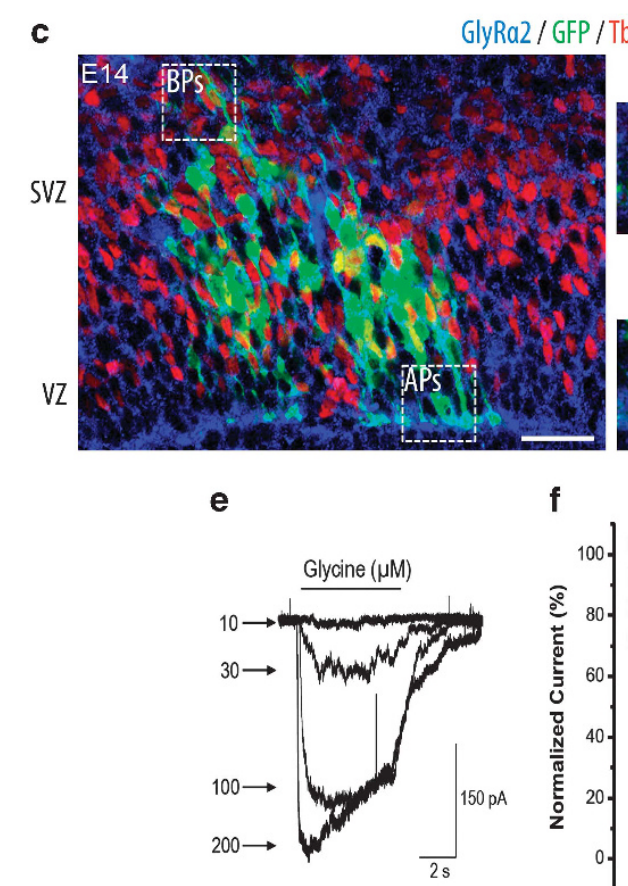

GlyRa2 / GFP / Tbr2

d

h

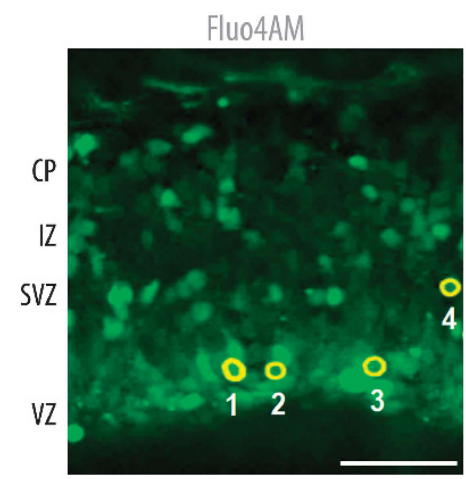

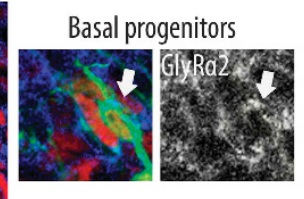

Apical progenitors

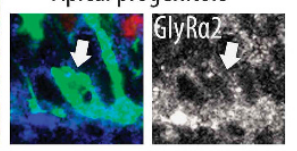

\section{f}
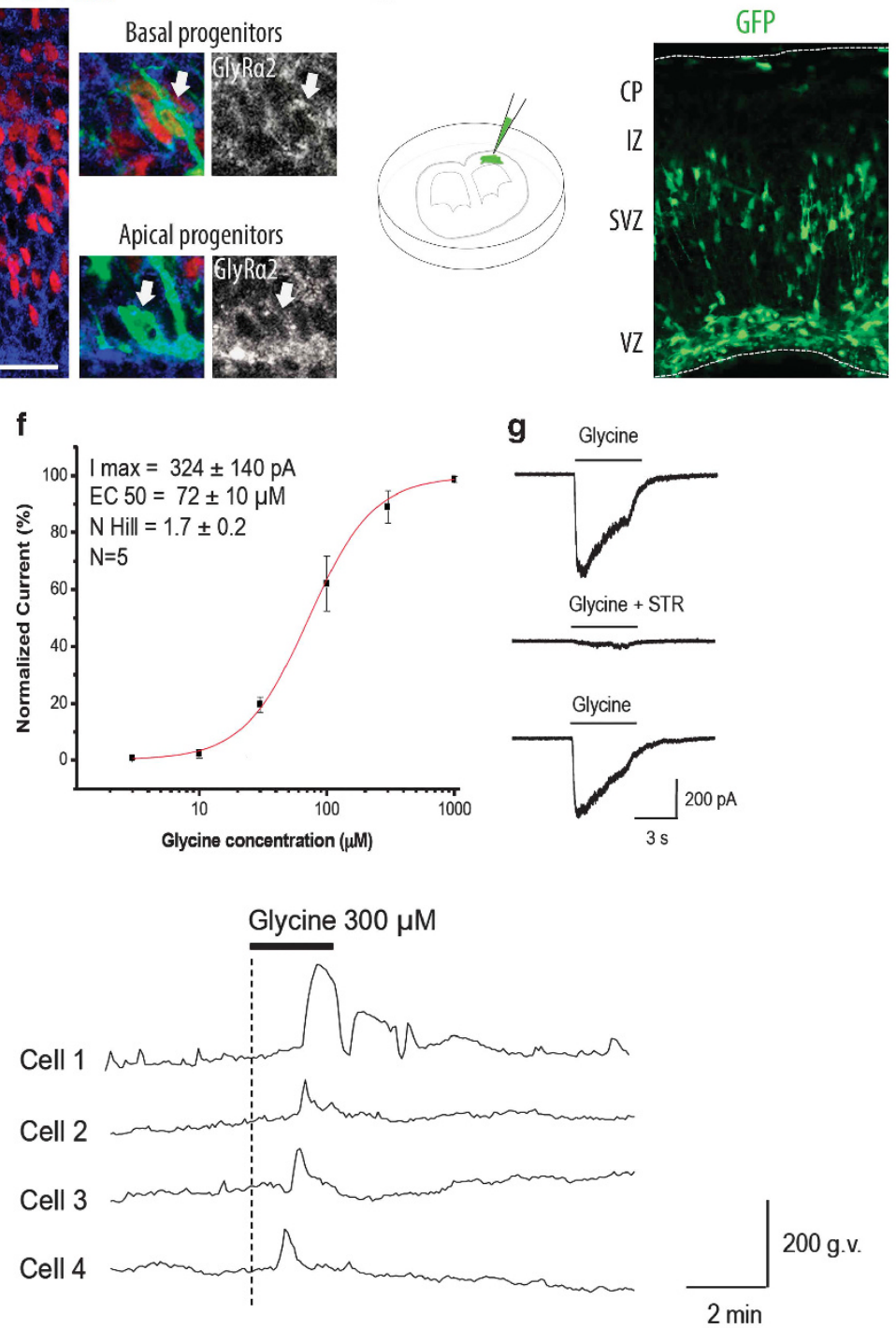
interneurons express GlyR $\alpha 2$ homomers that, when activated, promote neuronal migration during cortical development. ${ }^{32}$ Consistent with this finding, lack of Glra2 expression led to a marked reduction in cortical interneuron number at birth (Figures $2 \mathrm{~g}$ and $\mathrm{h}$ ). However, this neuronal population corresponds to a minor fraction of the cortical neurons. ${ }^{2,47}$ Therefore, depletion of interneurons alone was unlikely to account for the significant reduction of cortical wall thickness observed in newborn Glra2-KO mice. Given that functional GlyRs containing $\alpha 2$ are expressed by dorsal cortical progenitors (Figures $1 \mathrm{c}$ and d), we characterized their contribution to the generation of projection neurons during corticogenesis. The reduced thickness of the cortex of newborn Glra2-KO mice could result from either poor survival of newborn neurons and their progenitors or a reduction of neuronal output from these progenitors. Given the lack of significant changes in cortical cell survival in Glra2-KO cortices as compared with control (similar number of activated caspase-3 positive cells; data not shown), we assessed the proliferation rate of cortical progenitors, which if disrupted would affect the number of projection neurons destined for the cortical layers. Analyses conducted by immunohistochemistry on brain sections from wild-type (WT) or Glra2-KO E13 embryos revealed a significant reduction of $V Z$ and SVZ cycling progenitors that express Ki67 (Figure 2i). These results suggested the existence of early progenitor defects that could underlie microcephaly in newborn Glra2-KO mice.

Genetic inactivation of Glra2 disrupts cortical progenitor homeostasis. Our experiments revealed a reduced percentage of Ki67-positive cycling progenitors (Figure 2i) together with a specific depletion of Tbr2-positive BPs but not Pax6positive APs in Glra2 $\mathrm{KO}$ embryos, as compared with controls (Figures $3 a-d)$. The analysis performed on older embryos (E15) confirmed a significant reduction of Tbr2expressing cells and also revealed a progressive depletion of Pax6-expressing APs (Figures $3 g-j$ ). Surprisingly, the percentage of cortical progenitors undergoing mitosis (as revealed by $\mathrm{pHH} 3$ staining) increased in both E13 and E15 Glra2-KO cortices despite their reduced absolute number (Figures $3 e$ and $f$, and $k$ and $l$ ). This mitotic phenotype was indeed restricted in absolute cell number to APs in Glra2-KO cortices (Figures $4 a-d)$ ). It is noteworthy that APs undergo proliferative division to self-renew during early steps of corticogenesis, a cellular behavior characterized by mitotic cleavage plane orientation (Figure $4 \mathrm{e}$ ) close to $90^{\circ}$ (orthogonal to the ventricular surface). As corticogenesis proceeds, more neurons are generated through asymmetric divsions of APs that undergo mitosis with cleavage planes that have shorter angles. ${ }^{48}$ These cells generate neurons through direct or, in most cases, indirect neurogenesis via production of BPs. While the great majority of APs divided with mitotic cleavage plane angles ranging from $75^{\circ}$ to $90^{\circ}$ (Figures 4e-f, h) in E13 WT embryos, the analysis of Glra2KO cortices revealed over $50 \%$ of APs displaying angles between $30^{\circ}$ and $75^{\circ}$ (Figures $4 \mathrm{e}, \mathrm{g}$ and $\mathrm{h}$ ). These results demonstrate that lack of GlyR $\alpha 2$ expression leads to spindle orientation defects in APs. Importantly, this phenotype correlated with the reduced ability of APs to generate BPs (Tbr2 + in the SVZ) at the expense of neurons (Tbr2- in the IZ/CP) during corticogenesis, as shown by in utero electroporation of GFP in WT and Glra2 KO embryos (Figures 5ad). Thus, by bypassing the generation of BPs, which act as transit-amplifying progenitors for generation of projection neurons, Glra2-KO embryos likely have reduced neuronal output in the cortical wall, as previously reported after depletion of huntingtin in cortical APs. ${ }^{49}$

Genetic inactivation of Glra2 leads to depletion of cortical projection neurons. The reduced cortical wall thickness observed in E13 Glra2 KO embryos (E13) was also evident at birth (Figure 2a). The depletion of BPs in the cortical wall of Glra2 KO embryos suggested that microcephaly could result from an overall reduction of projection neuron output in the cortical wall. In order to address this hypothesis, we assessed cortical progenitor cell cycle exit and showed that this process was increased in the cortex of Glra2-knockout embryos at E13 (Figures 6a-c) but not earlier (E12, data not shown). However, the population of Tbr2 progenitors that initiated neuronal differentiation (co-expressing Tbr1) almost doubled at E12 in Glra2-KO embryos, as compared with WT controls (Figures 6d-f) and without significant differences for their individual expression (Figures $6 \mathrm{~g}$ and $\mathrm{h}$ ). Collectively, these results suggest a precocious differentiation of BPs into neurons as early as E12 in the cortical wall of Glra2 embryos.

We reasoned that a combination of increased cell-cycle exit with accelerated neuronal differentiation would progressively exhaust the pool of cortical progenitor, thus secondarily reduce the net overall production of projection neurons. In order to test this hypothesis, we assessed cortical layer markers in the cortex of newborn mice. In situ hybridization experiments revealed a reduced thickness of layers II to $\mathrm{V}$ in P0 Glra2-knockout embryos, as compared with control (Figures $7 \mathrm{a}-\mathrm{c}$ ). However, no clear differences were noticed between genotypes for layer VI neurons that express Tbr1 (Figure 7d). These results were further confirmed by immunolabelings using antibodies that recognize neurons

Figure 1 Expression of functional GlyRs during embryonic cortical development. (a, b) Immunolabelings performed on a brain section from WT or Glra2 KO (a, left and right, respectively) E13 embryos showing GlyR $\alpha 2$-subunit immunostaining in the cortical wall (GlyR $\alpha 2$, red; arrows point strong signal in cells bordering the lateral ventricle and asterisks show non-specific staining in $\mathbf{a}$. The expression pattern of GlyR $\alpha 2$ changes during development (b); nuclei are stained in blue with Dapi). (c, d) Apical and basal progenitors express GlyR $\alpha 2$-subunit (GlyR $\alpha 2$ in blue or white, Tbr2 in red and GFP in green). Arrows point either an apical or a basal progenitor on single confocal plane (c). Expression of GFP (green) in cycling APs and BPs $24 \mathrm{~h}$ after retroviral infection in cultured slices (see scheme on the left, (d). (e-g) Whole-cell patch-clamp analyses performed on cycling APs and or BPs in acute E13 brain slices. Representative traces of glycine-elicited currents obtained in APs after bath application of successive increasing glycine concentrations (e). Concentration-response curve obtained in BPs from successive bath application of increasing glycine concentrations (f). Representative traces of strychnine (STR) inhibition of glycine-elicited currents obtained in a BP (g). (h) Intracellular $\mathrm{Ca}^{2+}$ oscillations recorded in the cortical wall of brain slices loaded with Fluo4 AM (green). Representative traces of $\mathrm{Ca}^{2+}$ oscillations in representative cells $(1-4$, circled in yellow in the corresponding picture) induced by glycine application, as indicated. Scale bar, $65 \mu \mathrm{m}$ (a), $100 \mu \mathrm{m}$ (b), $25 \mu \mathrm{m}$ (c), $50 \mu \mathrm{m}$ (h). CP, cortical plate; IZ, intermediate zone; MZ, marginal zone; SVZ, subventricular zone; VZ, ventricular zone 

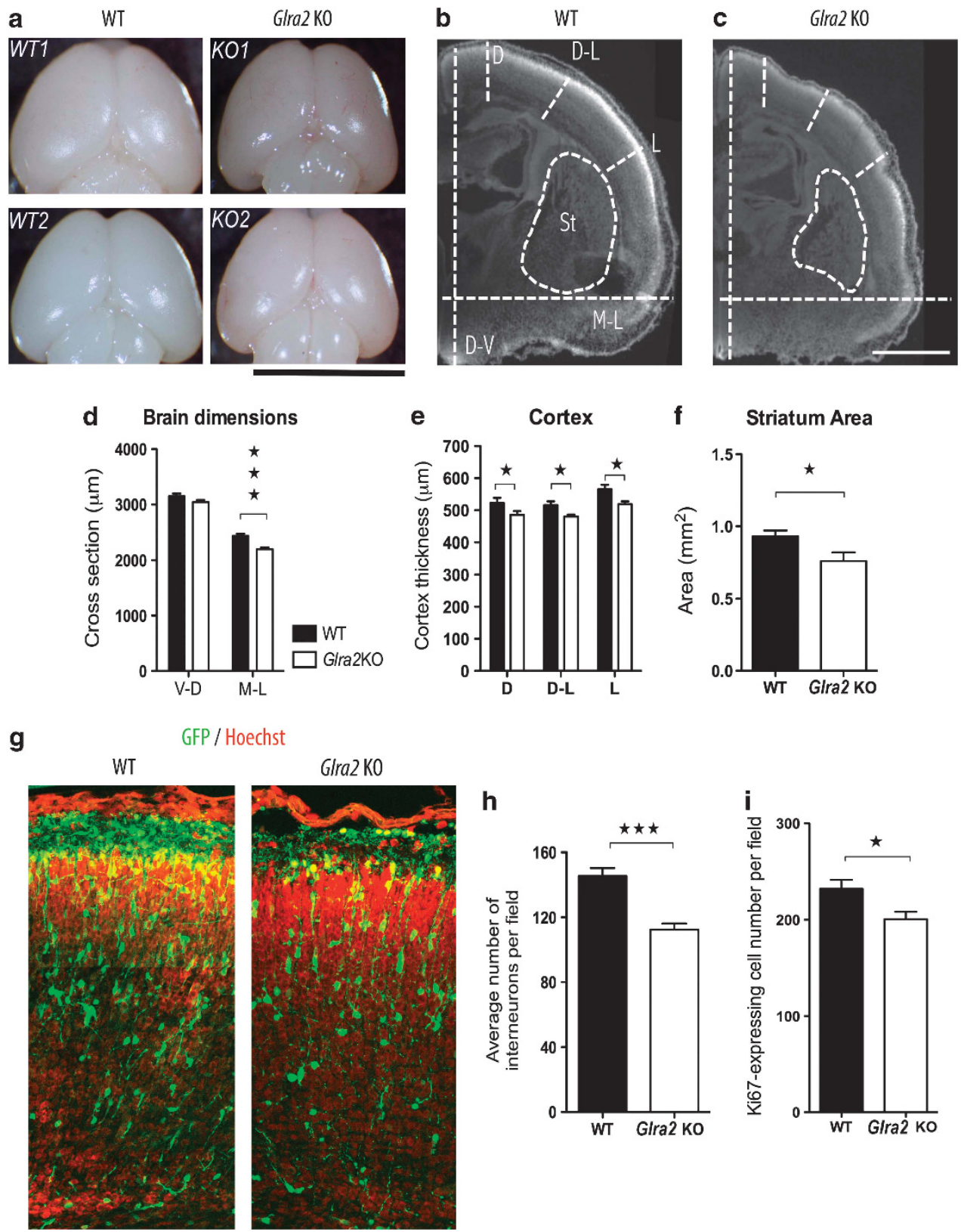

Figure 2 Genetic targeting of Glra2 impairs brain development. (a-f), newborn Glra2 KO mice have a smaller brain size, with reduced thickness of the cerebral cortex. Microphotography of brains from two distinct wild types (WT1, WT2) and two distinct Glra2 KO (KO1, KO2) newborn littermates at PO (a). Half coronal brain sections of wildtype (b), WT) or Glra2 knockout (c), Glra2 KO) newborn littermates. Histograms representing measurements obtained on brain sections from newborn WT or Glra2-KO mice (see (b, c) for representative sections) showing brain dimensions (d), thickness of the cerebral cortex at different dorso-lateral levels (e) and striatum areas (f). Values are expressed as mean \pm S.E.M., $n=10$ and 18 brain sections taken from three and five brains per genotype; ${ }^{*} P<0.05,{ }^{* * *} P<0.001$, $t$-test. (g-i) Immunolabelings showing cortical GFP-expressing interneurons (green) on brain sections from newborn WT or Glra2 KO littermates (g); nuclei are stained with Hoechst, red. Histogram of the average number of interneurons per field (h); mean \pm S.E.M., $n=6$ and 7 brain sections taken from three brains per genotype; ${ }^{* * \star} P<0.001, t$-test.). (i), number of VZ and SVZ cycling cells that express Ki67 (mean \pm S.E.M., $n=11$ and 14 brain sections taken from 3 brains per genotype; ${ }^{*} P<0.05$, $t$-test). Scale bar, $5 \mathrm{~mm}(\mathbf{a}), 1,000 \mu \mathrm{m}$ (c). D, dorsal; D-L, dorso-lateral; L, lateral; D-V, dorso-ventral; M-L, medio-lateral

that either settled into upper (layer II-IV, Cux1+) or deep (layer V, Ctip2 +; layer VI, Tbr1+) cortical layers. We observed a net depletion of upper layer neurons (Cux $1+$; Figures $7 \mathrm{e}$ and $\mathrm{g}$ ) in Glra2 $\mathrm{KO}$, as compared with WT littermates. Similar results were obtained for layer $\mathrm{V}$ neurons (Ctip2 + ; Figures $7 f$ and h) but not layer VI neurons (Tbr1 + ; Figures $7 f$ and i). Taken together, our results indicated that genetic invalidation of GlyRs in APs and BPs impairs their ability to proliferate and differentiate, and result in an overall reduction of neurogenesis in the developing cortical wall.

\section{Discussion}

Mammalian cerebral cortex neurogenesis is a tightly regulated process that requires establishment of a complex progenitor hierarchy. The complexity of cortical progenitors 

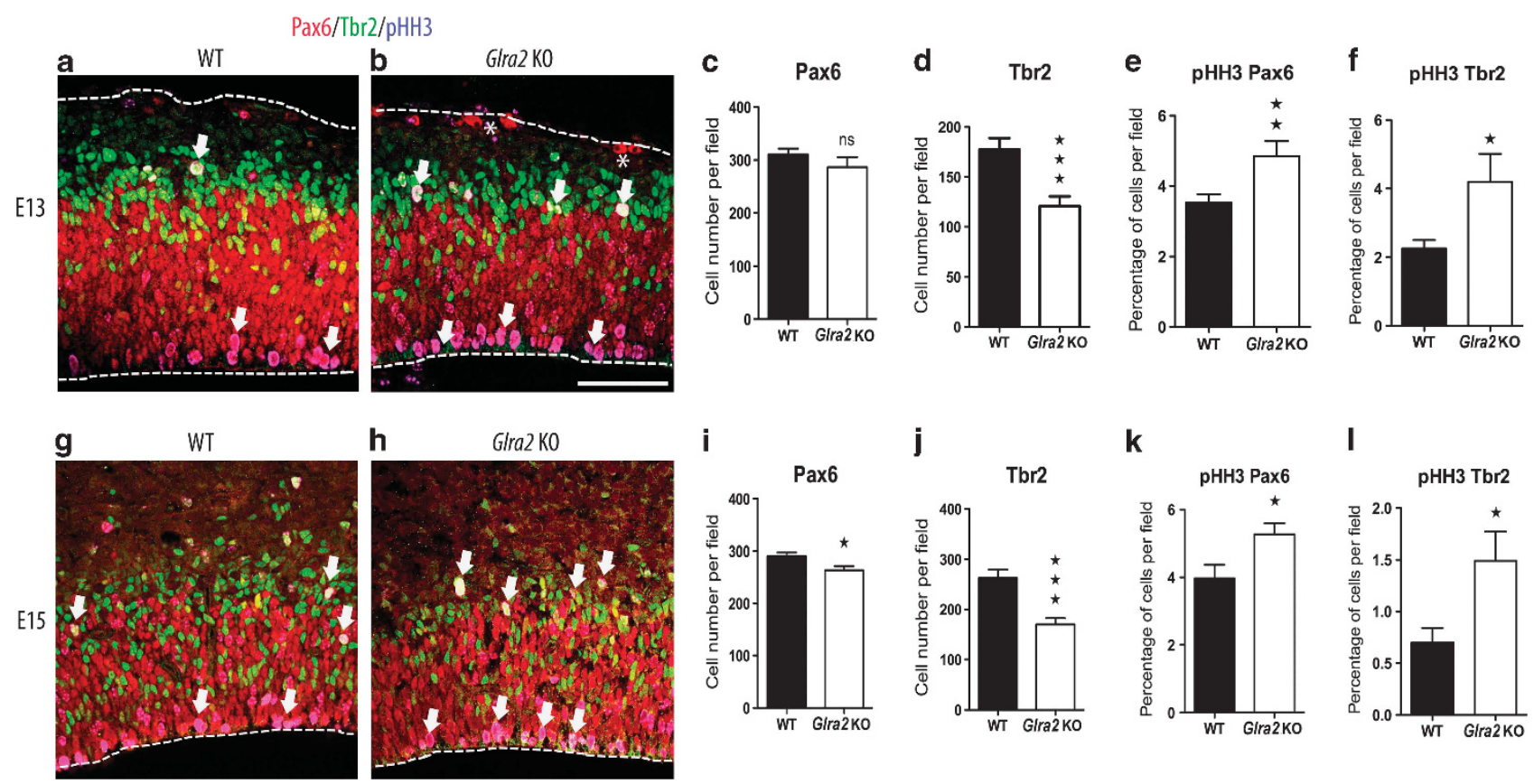

Figure 3 Genetic invalidation of Glra2 disrupts cortical progenitor homeostasis. (a, b) Immunolabelings showing Pax6-expressing APs (red), Tbr2-expreessing BPs (green) and progenitors undergoing mitosis that are positive for phospho-histone $\mathrm{H} 3$ (pHH3, blue) in brain sections from WT (a) or Glra2 KO (b) E13 embryos. (c-f) Histograms representing numbers of Pax6-postive APs per field (c), Tbr2-positive BPs per field (d), percentage of APs undergoing mitosis per field (Pax6- and pHH3-positives, (e) and percentage of BPs undergoing mitosis per field (Tbr2- and pHH3-positives, $\mathbf{f}$ ). Values are expressed as mean \pm S.E.M., $n=14$ and 16 brain sections taken from four brains per genotype; ${ }^{* *} P<0.01,{ }^{* \star *} P<0.001$, $t$-test (a-f). (g-l) Immunolabelings showing Pax6-expressing APs (red), Tbr2-expressing BPs (green) and progenitors undergoing mitosis that are positive for phospho-histone $\mathrm{H3}$ (pHH3, blue) in brain sections from WT (g) or Glra2KO (h) E15 embryos. (i-I) Histograms representing numbers of Pax6postive APs per field (i), Tbr2-positive BPs per field (j), percentage of APs undergoing mitosis per field (Pax6- and pHH3-positives, (k) and percentage BPs undergoing mitosis per field (Tbr2- and pHH3-positives, I). Values are expressed as mean \pm S.E.M., $n=16$ brain sections taken from four brains per genotype; ${ }^{\star} P<0.05$, ${ }^{* \star} P<0.01$, ${ }^{* \star *} P<0.001$, T-test. (g-l). Scale bar, $50 \mu \mathrm{m}$ (b). Arrows show pHH3-expressing cells $(\mathbf{a}, \mathbf{b}, \mathbf{g}, \mathbf{h})$

reflects the great variety of projection neuron phenotypes that settle later in bona fide cortical layers. ${ }^{50}$ At late stages of corticogenesis in mice, APs typically divide asymmetrically to generate either projection neurons or BPs that migrate in the $\mathrm{CP}$ or the SVZ, respectively. Basal progenitors act as transient-amplifying progenitors and generate the bulk of projection neurons destined for all cortical layers. ${ }^{42}$ The analyses performed on Glra2-KO embryos revealed moderate microcephaly, with depletion of projection neurons in upper and deep cortical layers. The results obtained with Glra2-KO support the following process: (1)APs generate less BPs at the expense of neurons and thus favor direct neurogenesis; (2) the overall reduced number of upper and deep layer neurons arises from significant depletion of BPs, as well as their accelerated differentiation into neurons; (3) the depletion of cortical neurons observed in Glra2-KO embryos leads to microcephaly (Figure 8).

Functional GlyRs are required for cortical progenitor homeostasis. GlyRs containing $\alpha 1$ and $\alpha 3$ subunits are expressed in spinal cord and brainstem circuits implicated in startle disease, inflammatory pain and rhythmic breathing. ${ }^{51}$ Although GlyRs are expressed in the hippocampus and cortex, ${ }^{21-27}$ their role in development has been largely overlooked. Over the past few years, we and others have discovered that GlyRs are highly expressed by specific neurons and progenitors of the cerebral cortex where they control critical developmental features. ${ }^{19}$ Two decades ago, pioneering studies described the expression of GlyR subunit mRNAs in the developing brain. In particular, GlyR $\alpha 2$ subunit transcripts were detected from E14 onwards in all cortical layers in rat embryos, ${ }^{33}$ and membrane currents triggered by GlyRs were recorded on migrating projection neurons in E19 rat embryos. ${ }^{34}$ These findings were further extended by experimental evidence supporting the existence of functional GlyRs in neurons located in the CP and IZ, ${ }^{38,44,52,53}$ as well as in migrating cortical interneurons. ${ }^{32}$ Our present study demonstrates that $\alpha 2$-containing GlyRs are functionally expressed in APs and BPs located in the developing cortical wall. These findings are in agreement with our recent results supporting the functional expression of GlyR $\alpha 2$ homomers in migratory interneurons and their progenitors. ${ }^{32}$ Although these results are in line with the detection of GlyR $\alpha 2$ subunit mRNAs in the developing cortical wall, ${ }^{33}$ they contrast with previous work reporting the lack of glycine-elicited membrane currents in VZ progenitors of developing rat embryos. ${ }^{10,34}$ Such discrepancy may arise as a consequence of developmental stage and/or species differences. Remarkably, our electrophysiological recordings demonstrate that GlyRs are functionally expressed in APs and BPs where glycine-elicited currents trigger $\mathrm{Ca}^{2+}$ influxes that may contribute to control of the proliferation and specification of these cortical progenitors.

A new role for GlyR $\alpha 2$ in cortical neurogenesis. Accumulating evidence supports a role for glutamate and 


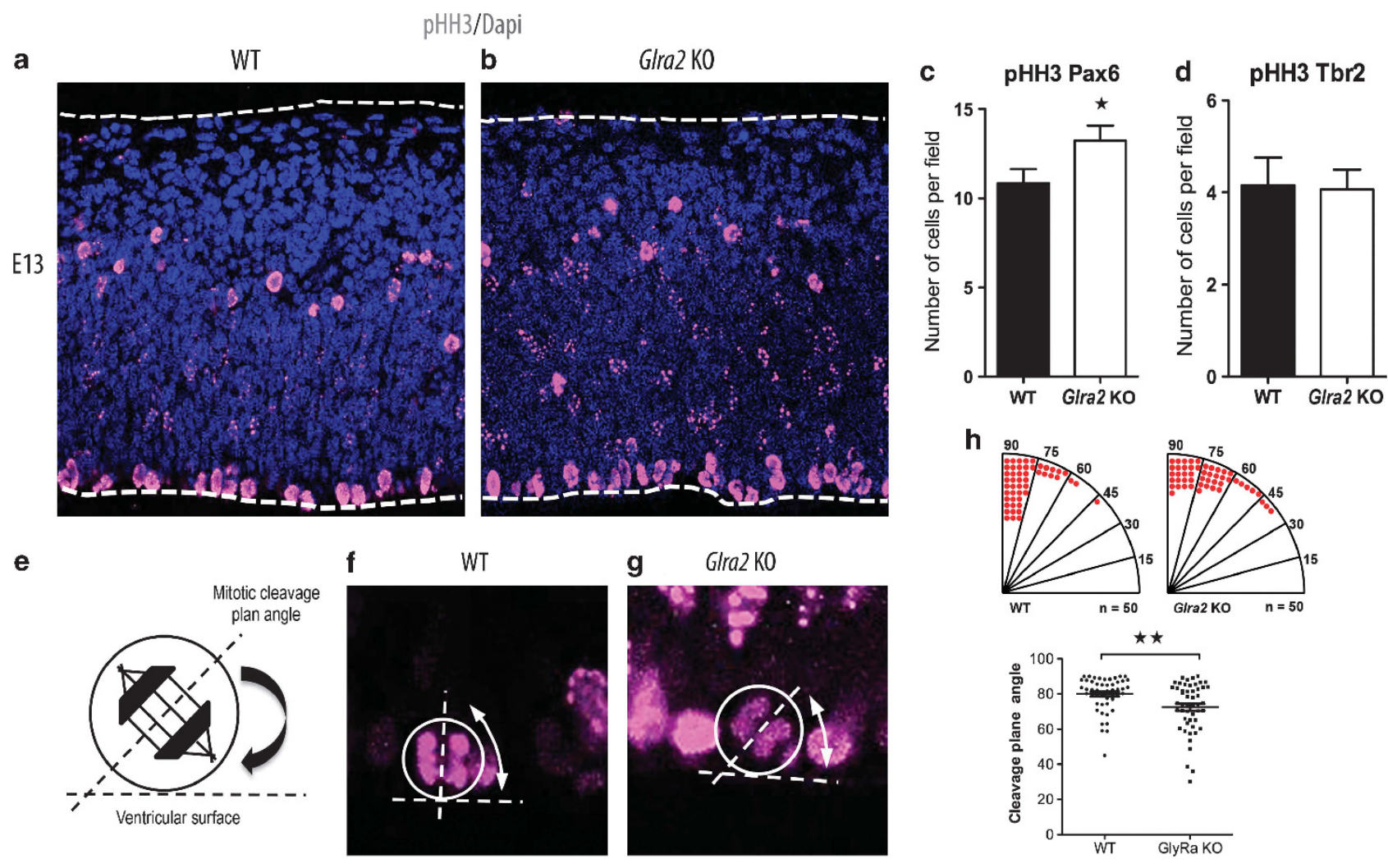

Figure 4 Genetic inactivation of Glra2 disrupts mitotic cleavage plane division. (a, b) Immunolabelings showing cells expressing phospho-histone H3 (pHH3, purple) and undergoing mitosis in the cortical wall of from WT (a) or Glra2 KO (b) E13 embryos. (c, d) Histograms representing absolute numbers of APs undergoing mitosis per field (Pax6- and pHH3-positives, (c) and absolute number of BPs undergoing mitosis per field (Tbr2- and pHH3-positives, d). Values are expressed as mean \pm S.E.M., $n=14$ and 16 brain sections taken from four brains per genotype; ${ }^{*} P<0.05$, $t$-test $(\mathbf{c}, \mathbf{d})$. (e-h) Scheme representing mitotic cleavage plane angle for APs (e), representative mitotic cleavage plane angles for APs undergoing mitosis in WT (f) or Glra2 KO E13 embryos. Distribution of mitotic cleavage plane angles of WT or Glra2 KO APs; ${ }^{* *} P<0.001$, Mann-Whitney test (h)

GABA in the control of neurogenesis. ${ }^{9,11}$ Indeed, endogenous activation of $\mathrm{GABA}_{A} \mathrm{Rs}$ controls several parameters of the cell cycle, including DNA synthesis ${ }^{10}$ and $\mathrm{G} 1$ phase duration. ${ }^{9}$ Interestingly, $\mathrm{GABA}_{\mathrm{A}} \mathrm{R}$ activation differentially affects progenitor cells located in the VZ and SVZ, ${ }^{9}$ supporting GABA as an extracellular molecular regulator of the equilibrium between APs and BPs. The present work adds another layer of complexity, by illustrating that glycineelicited currents control specific aspects of the biology of dorsal cortical progenitors. More specifically, loss of $\alpha 2$ containing GlyRs leads to an increased number of cortical APs undergoing mitosis, a result similar to observations made earlier in the developing spinal cord in response to morpholino-mediated knockdown of GlyR $\alpha 2$ subunits. ${ }^{54}$ Despite accumulation of ventricular mitosis in E15 Glra2$\mathrm{KO}$ embryos, the overall pool of APs was reduced, suggesting a progressive depletion of APs as a result of a premature shift from symmetric to asymmetric divisions. This hypothesis is supported by the demonstration that mitosis cleavage plane angles strongly deviated from the horizontal plane in GIra2-KO embryos, as compared with WT controls. Importantly, these results suggest that activation of GlyRs regulates the division mode of APs and thus contributes to cortical neurogenesis, a process that may indeed depend on the ability of GlyR $\alpha 2$-mediated currents to modulate actomyosin contractility via regulation of VGCCs and $\mathrm{Ca}^{2+}$ influxes. $^{32}$ In addition to being reduced in number, the remaining BPs differentiated more rapidly into Tbr1-expressing neurons in the cortex of Glra2-KO embryos. As BPs give rise to most cortical projection neurons ${ }^{42}$ and contribute to the expansion of the cerebral cortex observed during the evolution of mammals, ${ }^{55}$ the lack of GlyR $\alpha 2$ expression at the early steps of neurogenesis is likely to underline gross morphological defects, such as the impaired cortical lamination and moderate microcephaly observed in newborn Glra2KO mice. Surprisingly, layer VI neurons are generated properly in the cortex of Glra2-KO embryos. We hypothesize that GlyR $\alpha 2$ activity is required in cortical progenitors to control the generation of the appropriate number of projection neurons dedicated to most cortical layer, excluding layer VI (Tbr1 + neurons at postnatal stage). Indeed, we found that cell cycle exit, and thus the production of newborn projection neurons is enhanced at E13, but not before when most layer VI neurons are born (data not shown). It is noteworthy that no cortical phenotype was reported in a distinct Glra2-KO mouse line maintained in a genetic background ${ }^{52}$ different from the one used in our study.

Lengthening of G1 phase has been associated with a preference for 'differentiative' over 'proliferative' divisions of cortical progenitors, ${ }^{56,57}$ and altering $\mathrm{G} 1$ length impairs the 

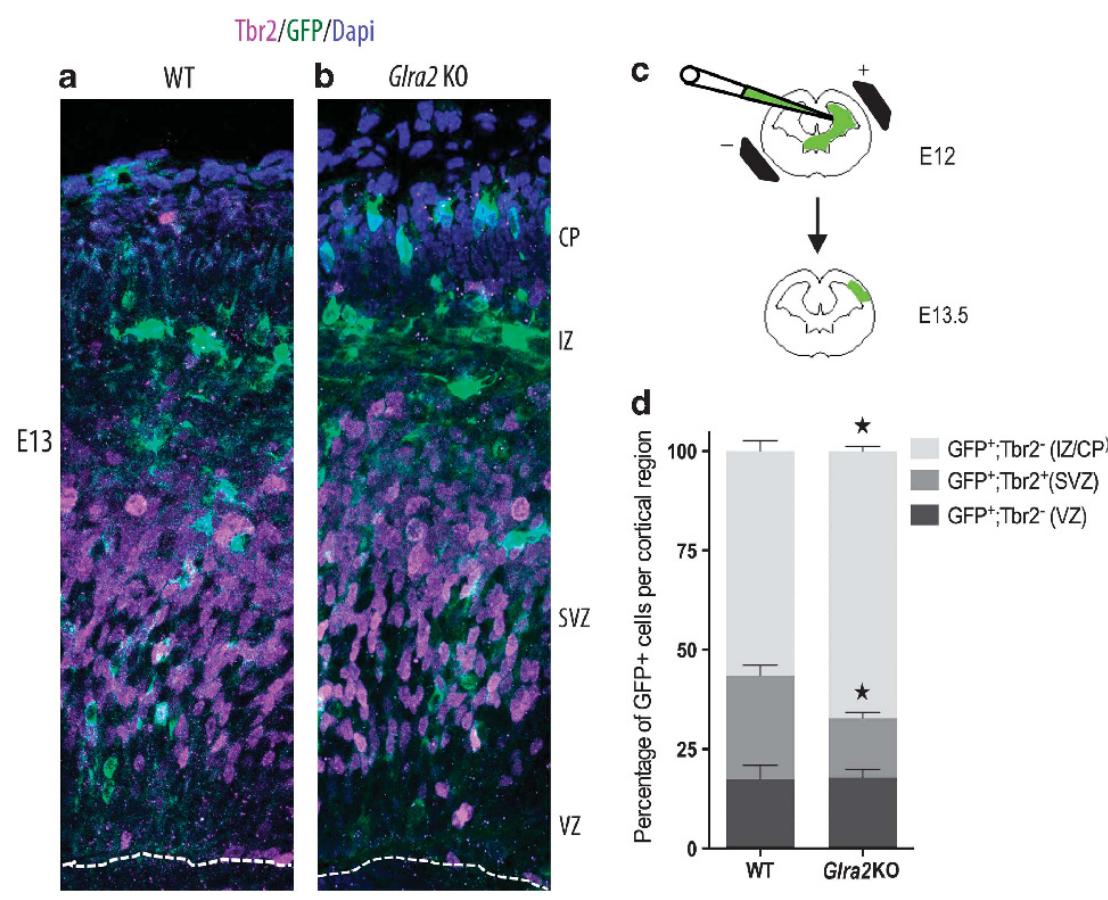

Figure 5 Genetic targeting of Glra2 impairs apico-basal transition in the developing cortex. (a, b) In utero electroporation of GFP-expressing plasmids was conducted on WT (a) or Glra2 KO (b) E13 embryos to target apical progenitors that lie against the ventricular membrane. Immunolabelings were performed $36 \mathrm{~h}$ later on brain sections from electroporated brain and show cells expressing Tbr2 (purple), GFP (green) and the nuclear label Dapi (blue). Scheme of the in utero electroporation procedure (c), see also material and methods). Histogram representing the percentage of electroporated cells (GFP + ) expressing or lacking Tbr2 in the ventricular zone (VZ), subventricular zone (SVZ) or intermediate zone and cortical plate (IZ and CP, respectively) (d); mean \pm S.E.M., $n=6$ and 8 brain sections taken from different brains per genotype; ${ }^{*} P<0.05, t$-test)

generation of appropriate number of projection neurons in the cerebral cortex. ${ }^{58,59}$ Disruption of $\mathrm{G} 1$ regulation may indeed characterize Glra2-KO APs and BPs, a defect that would further impact on the generation of proper projection neurons. This was supported by accumulation of immature neurons expressing both Tbr2 and Tbr1 as well as the progressive increase in cell cycle exit observed in Glra2 KO embryos, as compared with WT controls. Thus, expression of GlyR $\alpha 2$ by cortical progenitors seems to be required to prevent their premature specification/ differentiation into neurons and to support their self-renewal. The progressive developmental shift of GlyR $\alpha 2$ expression from germinal to postmitotic compartments (Figure 1b) may indeed reflect a progressive change in division mode of cortical progenitors that switch from 'proliferative' to 'differentiative' divisions as corticogenesis proceeds.

Contribution of extrasynaptic GlyRs to circuit development. Endogenous activation of GlyR cells autonomously controls interneuron migration during embryogenesis, ${ }^{32}$ and the present work extends these findings by showing their marked reduction in number in the cerebral cortex at birth. Remarkably, some interneurons have important role for the establishment of the first brain circuits. ${ }^{60,61}$ Moreover, appropriate development of cortical interneurons is required for proper activity of mature cortical circuits. ${ }^{62}$ Disruption of these circuits contributes to the pathophysiology of schizophrenia, autism and epilepsy. ${ }^{63-68}$ Our findings strongly suggest that GLRA2 should be shortlisted as a candidate gene in these disorders, as well as genetic studies of X-linked microcephaly.
Taken together, our study reveals a new developmental function for GlyR $\alpha 2$ in controlling the fate of progenitors of cortical projection neurons. We found that GIra2-KO mice exhibit a reduced brain size along with a reduced number of excitatory projection neurons in deep and upper layers of the cortex. These results indicate that the GlyRs expressed by cortical progenitors control the final number of excitatory projection neurons that later contribute to distinct mature brain circuits. Our work paves the way towards the identification of novel extracellular signals that, in concert with genetic programs, promote the establishment of mature neuronal circuits in the cerebral cortex.

\section{Materials and Methods}

Animals. All animal experiments were performed following guidelines of local ethical committees at Hasselt and Liège Universities. The mouse GlyR $\alpha 2$ subunit gene (Glra2) was selectively disrupted by homologous recombination using the Cre-LoxP gene targeting system (Kuhn et al., 1995) as previously described. ${ }^{32}$ Mice were genotyped by PCR. Glra2 knockouts were backcrossed onto the MF1 background, mated with Dlx5,6:Cre-IRES-EGFP (Dr. K Campbell, Cincinnati, USA) for selected experiments and housed at the BIOMED Institute animal facility.

Immunohistochemistry and RNA in situ hybridization. Embryonic brains were fixed by immersion in 4\% PFA for $30 \mathrm{~min}$ at E13, $1 \mathrm{~h}$ at E15 and $3 \mathrm{~h}$ at E17. The number of GFP + cortical interneurons was evaluated in WT and KO PO littermates born from breeding between DIx5,6:Cre-GFP and Glra2 + I - mice. For this purpose, mices were transcardiacally perfused with $4 \%$ PFA and their brains were post-fixed overnight in the same fixative. Brains were then cryoprotected overnight in $30 \%$ sucrose dissolved in PBS and processed to obtain $20 \mu \mathrm{m}$ cryosections. Bromodeoxyuridine (BrdU) labelings were performed as follows. Sections were washed twice for $5 \mathrm{~min}$ in PBS for rehydration of the samples. DNA denaturation and antigen retrieval were performed by incubation of 


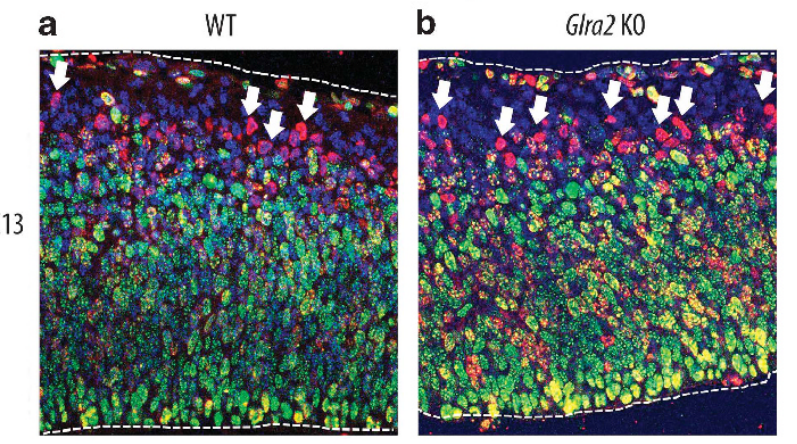

c

Tbr1/Tbr2/Dapi
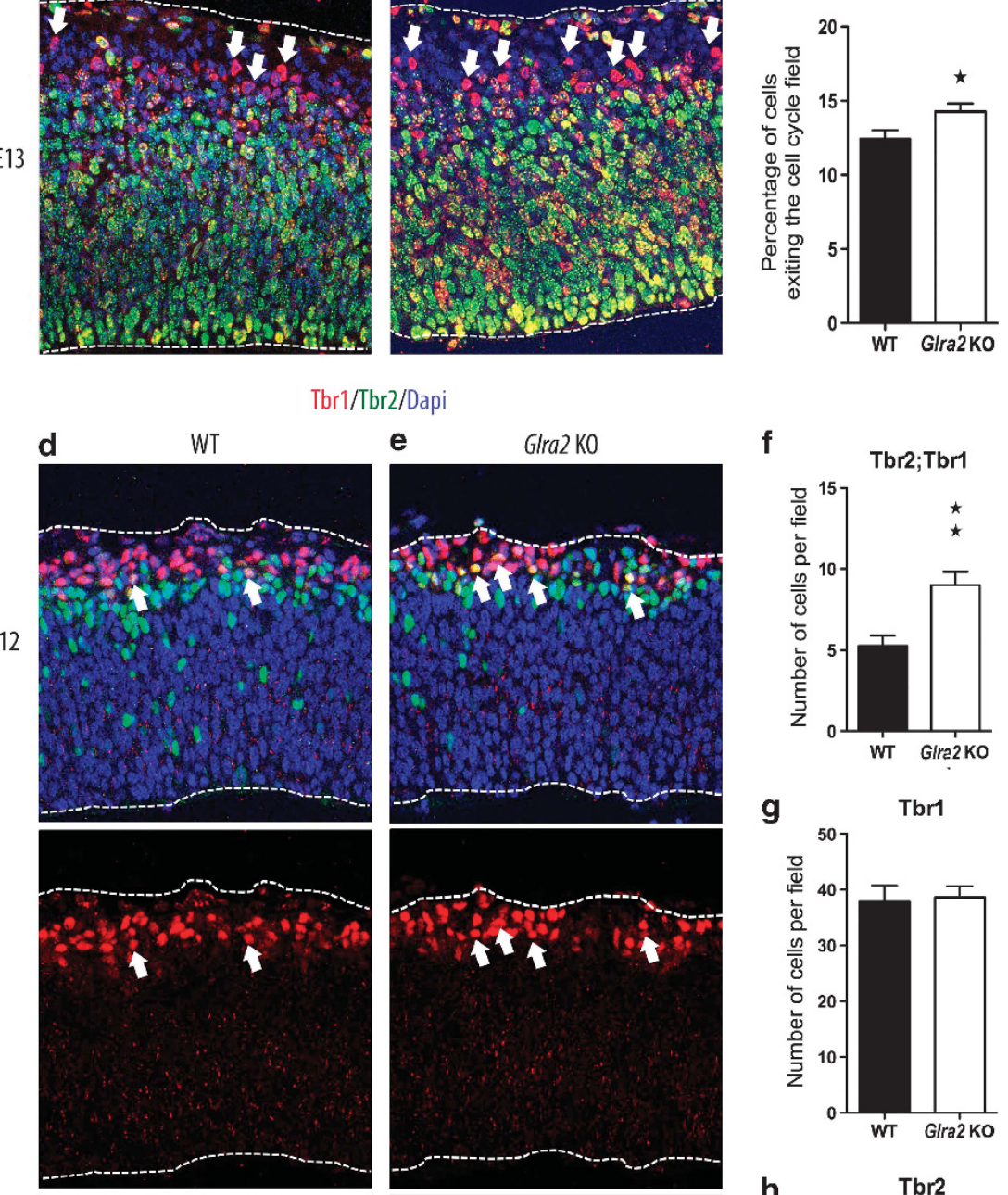

g
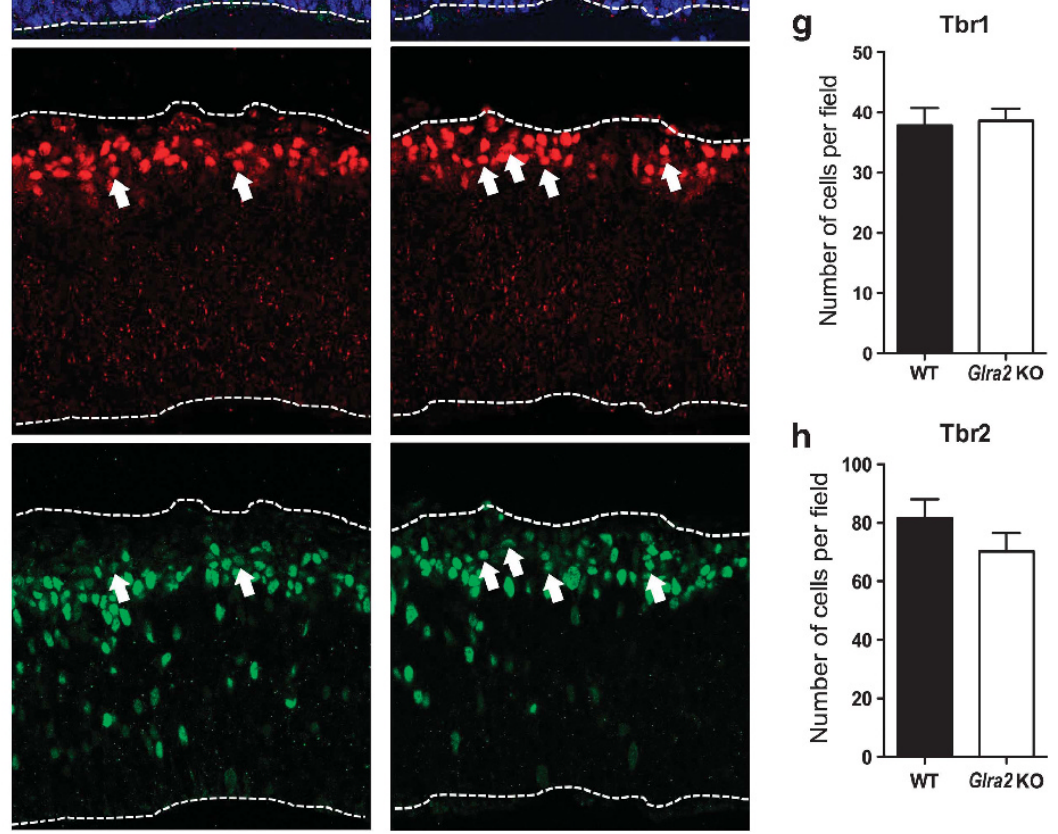

Figure 6 Genetic targeting of Glra2 impairs cell cycle exit and neuronal differentiation in the developing cortex. (a, b), Immunolabelings performed on brain section showing cells expressing BrdU (red), Ki67 (green) and the nuclear label Dapi (blue) in WT (a) or Glra2-KO (b) E13 embryos. Percentage of cells that exit the cell cycle per field (c; BrdU + /Ki67- cells; mean \pm S.E.M., $n=11$ and 15 brain sections taken from three and four brains for WT and Glra2 KO embryos, respectively). (d, e) Immunolabelings performed on brain section showing cells expressing Tbr1 (red), Tbr2 (green) and the nuclear label Dapi (blue) in WT (d) or Glra2 KO (e) E12 embryos. (f-h), histograms representing number of cells co-labeled for Tbr1 and Tbr2, per field (f), the number of cells expressing Tbr1 per field $(\mathbf{g})$ and the number of cell expressing Tbr2 per field ( $\mathbf{h}$ ). Values are expressed as mean \pm S.E.M., $n=16$ brain sections taken from four brains per genotype $(\mathbf{f}-\mathbf{h}) ;{ }^{*} P<0.05,{ }^{* *} P<0.01, t$-test

the slides in citrate buffer for $20 \mathrm{~min}$ at $100^{\circ} \mathrm{C}$. Following denaturation, slides were washed three times for $5 \mathrm{~min}$ in PBS. Blocking non-specific binding was performed by incubating slides $1 \mathrm{~h}$ in PBS containing $10 \%$ normal donkey serum (NDS, Tremecula) and $0.2 \%$ Triton X-100 (Sigma, Bornem, Belgium). The primary antiBrdU antibody (IgG1 BMC 2318 clone, Roche diagnostics GmbH, Mannheim, Germany) was diluted at 1:200 in PBS, and slices were incubated in this solution overnight. Secondary donkey anti-mouse Alexa 555 or 488 (A555 or A488, Life
Technologies, Gent, Belgium) dissolved in blocking solution was used at 1:500 for $1 \mathrm{~h}$ at room temperature. Slides were mounted with DAPI-containing mounting medium (Vector Laboratories, Burlingame, CA, USA) for counterstaining of nuclei. Ki67, Pax6, Tbr2 and pHH3 antigens as well as Tbr1, Ctip2 and Cux1 neuronal markers were detected applying a similar protocol using the following antibodies: rabbit anti-Ki67 (1:200; ab15580, Abcam, Cambridge, UK), mouse anti-Pax6 (1:10; PAX6-s, Developmental Studies Hybridoma Bank, lowa, IA, USA), Chicken 
a

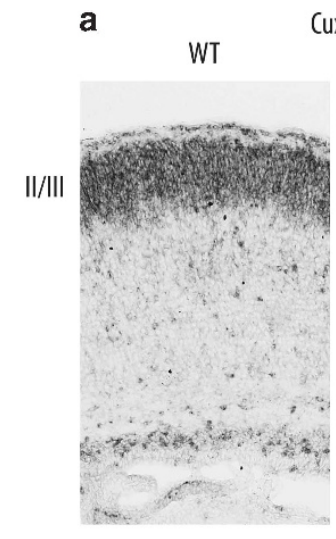

C

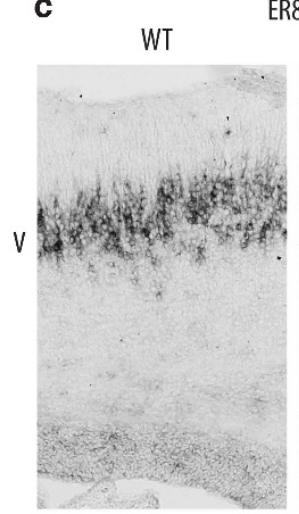

ER81

Glra2 KO

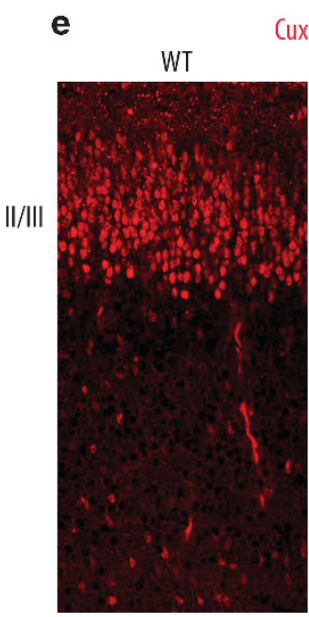

Cux1

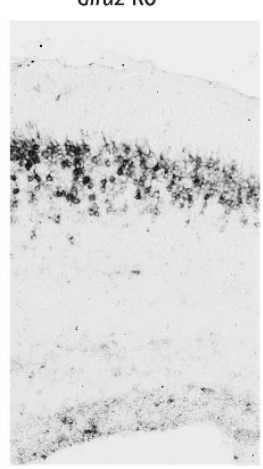

Glra2 KO
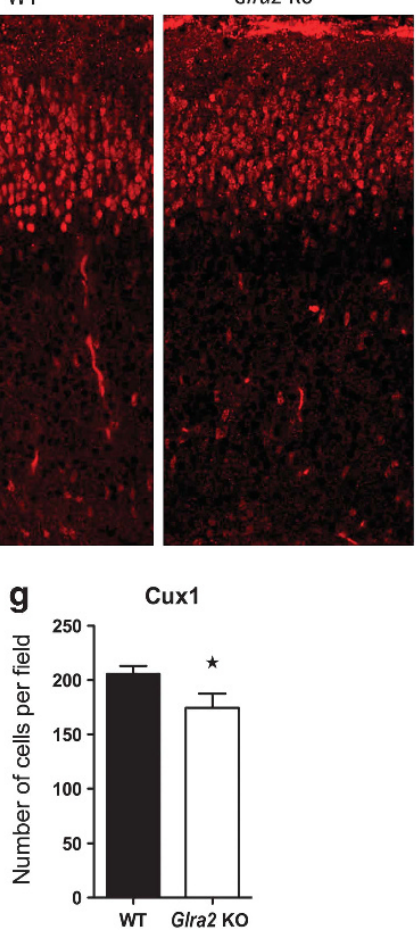

b

WT ROR Glra2 KO

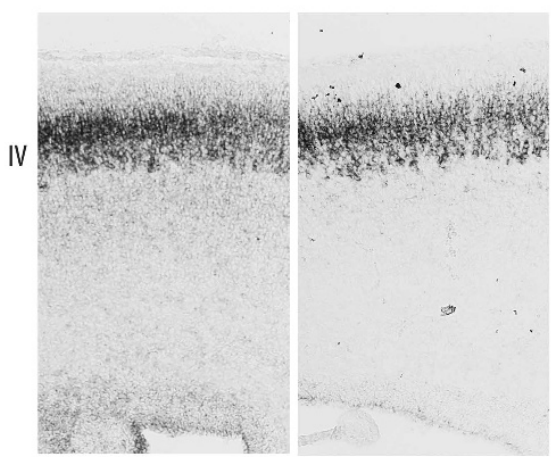

d

$\begin{array}{lll}\text { WT } & \text { Tbr1 } & \text { KO }\end{array}$

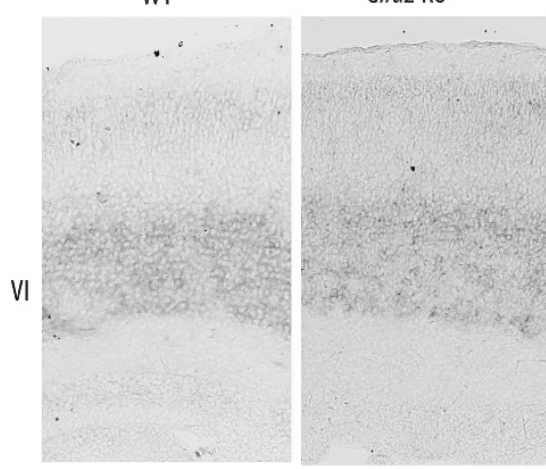

f

Ctip2/Tbr1/Dapi

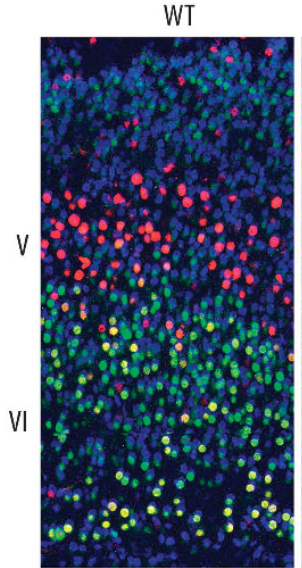

Glra2 KO

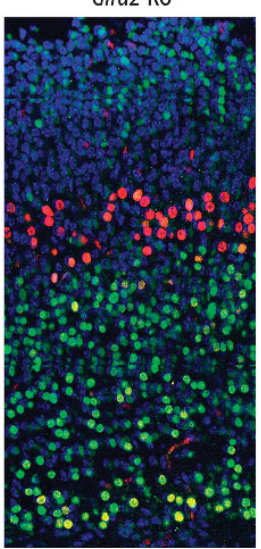

h

Ctip2

i

Tbr1
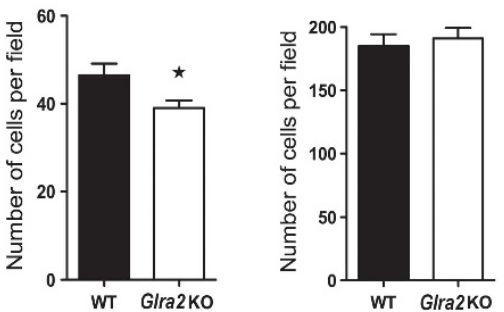

Figure 7 Genetic targeting of Glra2 results in a reduced number of projection neurons in upper and deep cortical layers. (a-d) mRNA labeling after in situ hybridization in brain sections from PO WT or Glra2 KO mice. Latin numbers indicate cortical layer identity. (e-i) Immunolabelings performed on brain section showing cells expressing Cux1 (red, (e), Ctip2 (red, (f) Tbr1 (green, (f) and the nuclear label Dapi (blue) in PO WT or Glra2 KO mice. ( $g$-i) Histograms representing the number of Cux1-expressing neurons per field $(\mathbf{g})$, the number of Ctip2-expressing neurons per field $(\mathrm{h})$ and the number of Tbr1-expressing neurons per field (i); mean \pm S.E.M., $n=10$ brain sections taken from three brains per genotype; ${ }^{*} P<0.05, t$-test). $\left(\mathrm{g}\right.$-i) Values are expressed as mean \pm S.E.M., $n=11$ brain sections taken from three brains per genotype; ${ }^{*} P<0.05, t$-test 
WT

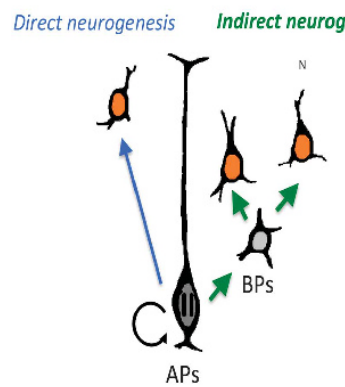

Glra2 K0 Direct neurogenesis Indirect neurogenesis

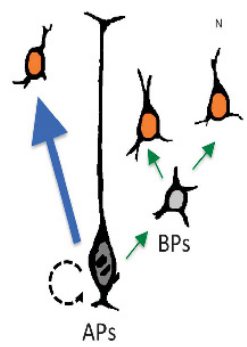

Figure 8 Genetic targeting of G/ra2 impairs neuron generation by affecting the homeostasis of cortical progenitors, a model. Summary scheme illustrating cortical progenitor division and neurogenesis in the developing cerebral cortex of WT or Glra2-KO embryos.

anti-Tbr2 (1:200; AB15894, Millipore, Overijse, Belgium), Rabbit anti-pHH3 (1:200; 06-570, Millipore), Rabbit anti-Tbr1 (1:200; ab31940, Abcam), rat anti-Ctip2 (1:200; ab18465, Abcam) and goat anti-Cux1 (1:50; Sc-6327, Santa Cruz Biotechnologies, Heidelberg, Germany). All antibodies were incubated in blocking solution. Double and triple labelings were performed by one step co-incubation with a mixture of the appropriate antibodies. The same strategy was used for incubation with the secondary antibodies (Alexa, 1:500; Life Technologies). Pax6 was better observed when replacing PBS by TBS during immunolabelings. As an exception, for the detection of GlyRs, brain sections were washed with $\mathrm{NH}_{4} \mathrm{Cl}$ and then with PBS for 30 min. Blocking was made in 10\% NDS plus 1\% BSA dissolved in PBS for $1 \mathrm{~h}$ at room temperature. GlyR detection was performed using the $\alpha 2$ specific antibody N18 (1:100; Sc-17279, Santa Cruz Biotechnologies) diluted in PBS and containing $3 \%$ NDS plus $1 \%$ BSA in PBS, overnight at $4{ }^{\circ} \mathrm{C}$. The secondary antibody was a donkey anti-goat labeled with A647 (1:500; Life Technologies). GFP and Tbr2 double labeling also required special conditions. In this case, GFP was detected using rabbit anti-GFP (1:200; ab6556, Abcam). Samples were further incubated overnight with antibodies and then fixed for $15 \mathrm{~min}$ at $-20^{\circ} \mathrm{C}$ in acetone. Sections were washed in PBS-tween and incubated in $95^{\circ} \mathrm{C}$ citrate buffer for $2-5 \mathrm{~min}$, then washed with PBS-T followed by a second blocking step. At this point, the Tbr2 antibody was incubated overnight to finish with an incubation using a mixture of goat anti-rabbit A488 and goat-anti chicken A647 (1:500; Life Technologies) for the detection of GFP and Tbr2, respectively. The number of cortical interneurons was evaluated in WT and KO PO littermates born from breeding between DIx5,6:Cre-GFP and Glra2 + I - mice. RNA in situ hybridizations were performed on frozen brain sections with 3 -digoxigenin-labeled antisense probes, as described previously, ${ }^{69}$ to Cux2, RoR $\beta$, ER81, Tbr1 (plasmids were given by François Guilllemot, NIMR, London, UK).

Confocal imaging. Images of labeled brain sections were acquired with a Carl Zeiss Axiovert $200 \mathrm{M}$ motorized microscope equipped with a LSM 510 META confocal laser scanner system (Carl Zeiss, Zaventem, Belgium). For cell counting purposes, images were acquired with a Plan Apochromat $20 \times 10.75$ or PlanNeofluar $40 \times / 1.3$ Oil DIC objectives. The A488 fluorophore was visualized by excitation with the $488 \mathrm{~nm}$ emission line of an Ar-lon laser, while the A555 and A647 dyes were visualized using the $543 \mathrm{~nm}$ spectral line of the He-Ne laser and the $633 \mathrm{~nm}$ spectral line of a second He-Ne laser, respectively. DAPI excitation was achieved by two-photon excitation at $750 \mathrm{~nm}$ with the light emitted by a mode locked MaiTai laser (Spectra Physics, Irvine, CA, USA). Z-stacks were acquired every $1.5 \mu \mathrm{m}$. For sections older than E13, more than one Z-stack was acquired to capture the whole thickness of the cortex covering the same distance in the VZ as compared with images taken at E12-E13.

Electrophysiology. Dorsal progenitors or radially migrating cells were labeled by infection of E13 slices with GFP encoding retroviruses, which labeled proliferating cells. Application of retroviruses was performed $4 \mathrm{~h}$ after slicing and was restricted to the ventricles. One microliter of the diluted viral suspension was applied to each hemisphere. Following this procedure, GFP-positive cells were already visible lining the ventricles after $24 \mathrm{~h}$. Whole-cell patch-clamp recordings were performed using an Axon 200B patch-clamp amplifier (Harvard Apparatus, Holliston, MA, USA). All experiments were carried out at room temperature, in voltage-clamp mode at a holding voltage equal to $-60 \mathrm{mV}$. The internal solution was composed of $\mathrm{KCl} 130, \mathrm{NaCl} 5, \mathrm{CaCl}_{2} 1, \mathrm{MgCl}_{2} 1$, Hepes 10, EGTA 10, NaATP 2 and NaGTP $0.5(\mathrm{mM})$, and the external ACSF was composed of $\mathrm{NaCl} 125, \mathrm{KCl} 2.5, \mathrm{MgCl}_{2} 1, \mathrm{CaCl}_{2} 2, \mathrm{NaHCO}_{3} 25, \mathrm{NaH}_{2} \mathrm{PO}_{4}$ 1.25, Glucose $25(\mathrm{mM})$. The amplitude of glycine-elicited currents was assessed by brief applications that lasted for $5 \mathrm{~s}$. All ligands and blockers were focally applied, at a distance around $150 \mu \mathrm{m}$ from the surface of the slice, using a Warner perfusion system that allowed an exchange time of $<20 \mathrm{~ms}$ (Fast-Step, Warner Instruments LLC, Hamden, CT, USA). Data acquisition and analysis were performed using pClamp and Clampfit software, respectively (Harvard Apparatus).

$\mathrm{Ca}^{2+}$ imaging. $\mathrm{Ca}^{2+}$ imaging was performed using Fluo 4 (Life Technologies) in slices. Fluo 4 was excited by using light of $820 \mathrm{~nm}$ delivered by a pulsed laser. Loading was performed at $37^{\circ} \mathrm{C}$ for $30 \mathrm{~min}$ in agitation without a holding insert. Responses evoked by glycine or GABA were recorded in ACSF at $37^{\circ} \mathrm{C}$. Agonist application was achieved by bath perfusion. Image intensity analyses were made using ImageJ software (NIH), defining regions of interest and analyzing the change of intensity with time. Numeric values for intensity were then converted into traces using Clampfit (Harvard Apparatus).

In utero electroporation. In brief, embryonic brains were injected in vivo in the lateral ventricles with plasmid DNA-encoding GFP driven by ubiquitous promoter (pCAGGS-GFP) or by BLBP promoter (BLBP-GFP, gift from Dr. Jamel Chelly Institut Cochin, Paris) and were electroporated (5 pulses of 38 Volts, lasting for $50 \mathrm{~ms}$ each, every $1 \mathrm{~s}$ ) using an ECM 830 square pulse electroporation device (Harvard Apparatus) at E12 or E13. The DNA concentration used was $1.4 \mu \mathrm{g} / \mu \mathrm{l}$. Successfully electroporated cells were identified by GFP expression. Embryos were fixed either at E13.5 or E14 by $1 \mathrm{~h}$ immersion in 4\% PFA and processed for immunolabeling.

Cell counting and statistics. All image quantifications were performed in the brightest single plane along the Z-stack acquired with a $\times 40$ objective as described above. Analysis of cell counts in the developing somatosensory cortex at different ages was carried out using the manual cell counter tool included ImageJ. The dimension of analyzed field was $225 \times 225 \mu \mathrm{m}^{2}$ for E12-E13 brain sections, while for older ages the $Y$ dimension was increased to fit the entire cortex from the ventricle to the pial surface keeping $225 \mu \mathrm{m}$ for $X$. All values are expressed as mean \pm S.E.M. Mean and error calculations, as well as statistical analyses, were computed in GraphPad (GraphPad Software, Inc., La Jolla, CA, USA).

\section{Conflict of Interest}

The authors declare no conflict of interest.

Acknowledgements. We thank Dr. F Guillemot (NIMR, London, UK) and Dr. Jamel Chelly (Institut Cochin, Paris, France) for providing plasmids, Dr. K Campbell (University of Cincinnati, USA) for providing the Dlx5,6:Cre-GFP mouse line, and Neil T. Dear (South Australian Health and Medical research Institute, Australia) for providing the Glra2-knockout mouse line. The antibody against Pax6 was developed by Dr. A Kawakami and was obtained from the Developmental Studies Hybridoma Bank developed under the auspices of the NICHD and maintained at The University of lowa, Department of Biology, lowa City, IA 52242. RJH is funded by the Medical Research Council (G0500833). LN is a Research Associate from the Belgian National Fund for Scientific Research (F.R.S- F.N.R.S.). LN is funded by grants from the F.R.S.-F.N.R.S., the Fonds Léon Fredericq, the Fondation Médicale Reine Elisabeth, the Belgian Science Policy (IAP-VII network P7/20), and the Actions de Recherche Concertées (ARC11/16-01). Some scientific projects in the Nguyen laboratory are funded by the Walloon Excellence in Life Sciences and Biotechnology (WELBIO).

1. Gupta A, Tsai LH, Wynshaw-Boris A. Life is a journey: a genetic look at neocortical development. Nat Rev Genet 2002; 3: 342-355.

2. Anderson SA, Eisenstat DD, Shi L, Rubenstein JL. Interneuron migration from basal forebrain to neocortex: dependence on Dlx genes. Science 1997; 278: 474-476.

3. Bielas S, Higginbotham H, Koizumi H, Tanaka T, Gleeson JG. Cortical neuronal migration mutants suggest separate but intersecting pathways. Annu Rev Cell Dev Biol 2004; 20: 593-618.

4. Casanova MF, Trippe J 2nd. Regulatory mechanisms of cortical laminar development. Brain Res Rev 2006; 51: 72-84. 
5. Represa A, Ben-Ari Y. Trophic actions of GABA on neuronal development. Trends Neurosci 2005; 28: 278-283.

6. Heng Jl, Moonen G, Nguyen L. Neurotransmitters regulate cell migration in the telencephalon. Eur J Neurosci 2007; 26: 537-546.

7. Nguyen L, Rigo JM, Rocher V, Belachew S, Malgrange B, Rogister B et al. Neurotransmitters as early signals for central nervous system development. Cell Tissue Res 2001; 305: 187-202

8. Soria JM, Valdeolmillos M. Receptor-activated calcium signals in tangentially migrating cortical cells. Cereb Cortex 2002; 12: 831-839.

9. Haydar TF, Wang F, Schwartz ML, Rakic P. Differential modulation of proliferation in the neocortical ventricular and subventricular zones. J Neurosci 2000; 20: 5764-5774.

10. LoTurco JJ, Owens DF, Heath MJ, Davis MB, Kriegstein AR. GABA and glutamate depolarize cortical progenitor cells and inhibit DNA synthesis. Neuron 1995; 15 1287-1298

11. Nguyen L, Malgrange B, Breuskin I, Bettendorff L, Moonen G, Belachew S et al. Autocrine paracrine activation of the $\mathrm{GABA}(\mathrm{A})$ receptor inhibits the proliferation of neurogenic polysialylated neural cell adhesion molecule-positive (PSA-NCAM +) precursor cells from postnatal striatum. J Neurosci 2003; 23: 3278-3294.

12. Bolteus AJ, Bordey A. GABA release and uptake regulate neuronal precursor migration in the postnatal subventricular zone. J Neurosci 2004; 24: 7623-7631.

13. Manent JB, Demarque $M$, Jorquera I, Pellegrino $C$, Ben-Ari $Y$, Aniksztejn $L$ et al. A noncanonical release of GABA and glutamate modulates neuronal migration. $J$ Neurosci 2005; $25:$ 4755-4765.

14. Cancedda L, Fiumelli $\mathrm{H}$, Chen $\mathrm{K}$, Poo MM. Excitatory GABA action is essential for morphological maturation of cortical neurons in vivo. I Neurosci 2007; 27: 5224-5235.

15. Rivera C, Voipio J, Payne JA, Ruusuvuori E, Lahtinen $\mathrm{H}$, Lamsa $\mathrm{K}$ et al. The $\mathrm{K}+/ \mathrm{Cl}$ co-transporter KCC2 renders GABA hyperpolarizing during neuronal maturation. Nature 1999; 397: 251-255.

16. Yamada J, Okabe A, Toyoda H, Kilb W, Luhmann HJ, Fukuda A. Cl- uptake promoting depolarizing GABA actions in immature rat neocortical neurones is mediated by NKCC1. J Physiol 2004; 557(Pt 3): 829-841.

17. Denter DG, Heck N, Riedemann T, White R, Kilb W, Luhmann HJ. GABAC receptors are functionally expressed in the intermediate zone and regulate radial migration in the embryonic mouse neocortex. Neuroscience 2010; 167: 124-134

18. Lynch JW. Native glycine receptor subtypes and their physiological roles. Neuropharmacology 2009; 56: 303-309.

19. Avila A, Nguyen L, Rigo JM. Glycine receptors and brain development. Front Cell Neurosc 2013; 7: 184.

20. Moss SJ, Smart TG. Constructing inhibitory synapses. Nat Rev Neurosci 2001; 2 240-250.

21. Zhang LH, Gong N, Fei D, Xu L, Xu TL. Glycine uptake regulates hippocampal network activity via glycine receptor-mediated tonic inhibition. Neuropsychopharmacology 2008; 33 701-711.

22. Aroeira RI, Ribeiro JA, Sebastiao AM, Valente CA. Age-related changes of glycine receptor at the rat hippocampus: from the embryo to the adult. $J$ Neurochem $2011 ; 118$ 339-353.

23. Song W, Chattipakorn SC, McMahon LL. Glycine-gated chloride channels depress synaptic transmission in rat hippocampus. J Neurophysiol 2006; 95: 2366-2379.

24. Keck T, Lillis KP, Zhou YD, White JA. Frequency-dependent glycinergic inhibition modulates plasticity in hippocampus. J Neurosci 2008; 28: 7359-7369.

25. Xu TL, Gong N. Glycine and glycine receptor signaling in hippocampal neurons: diversity, function and regulation. Prog Neurobiol 2010; 91: 349-361.

26. Sorensen SA, Bernard A, Menon V, Royall JJ, Glattfelder KJ, Hirokawa K et al. Correlated gene expression and target specificity demonstrate excitatory projection neuron diversity. Cereb Cortex 2013; e-pub ahead of print 19 December 2013; doi: 10.1093/cercor/ bht243.

27. Waseem TV, Fedorovich SV. Presynaptic glycine receptors influence plasma membrane potential and glutamate release. Neurochem Res 2010; 35: 1188-1195.

28. Turecek R, Trussell LO. Presynaptic glycine receptors enhance transmitter release at mammalian central synapse. Nature 2001; 411: 587-590.

29. Jeong HJ, Jang IS, Moorhouse AJ, Akaike N. Activation of presynaptic glycine receptors facilitates glycine release from presynaptic terminals synapsing onto rat spinal sacral dorsal commissural nucleus neurons. J Physiol 2003; 550(Pt 2): 373-383.

30. Xiong W, Chen SR, He L, Cheng K, Zhao YL, Chen H et al. Presynaptic glycine receptors as a potential therapeutic target for hyperekplexia disease. Nat Neurosci $2014 ; 17$ : 232-239.

31. Ye JH, Wang F, Krnjevic K, Wang W, Xiong ZG, Zhang J. Presynaptic glycine receptors on GABAergic terminals facilitate discharge of dopaminergic neurons in ventral tegmental area. J Neurosci 2004; 24: 8961-8974.

32. Avila A, Vidal PM, Dear TN, Harvey RJ, Rigo JM, Nguyen L. Glycine receptor alpha2 subunit activation promotes cortical interneuron migration. Cell Rep 2013; 4 738-750

33. Malosio ML, Marqueze-Pouey B, Kuhse J, Betz H. Widespread expression of glycine receptor subunit mRNAs in the adult and developing rat brain. EMBO $J 1991 ; 10$ 2401-2409.
34. Flint AC, Liu X, Kriegstein AR. Nonsynaptic glycine receptor activation during early neocortical development. Neuron 1998; 20: 43-53

35. Kilb W, Ikeda M, Uchida K, Okabe A, Fukuda A, Luhmann HJ. Depolarizing glycine responses in Cajal-Retzius cells of neonatal rat cerebral cortex. Neuroscience 2002; 112: 299-307.

36. Nguyen L, Malgrange B, Belachew S, Rogister B, Rocher V, Moonen G et al. Functional glycine receptors are expressed by postnatal nestin-positive neural stem/progenitor cells. Eur J Neurosci 2002; 15: 1299-1305.

37. Okabe A, Kilb W, Shimizu-Okabe C, Hanganu IL, Fukuda A, Luhmann HJ. Homogenous glycine receptor expression in cortical plate neurons and Cajal-Retzius cells of neonatal rat cerebral cortex. Neuroscience 2004; 123: 715-724.

38. Nimmervoll B, Denter DG, Sava I, Kilb W, Luhmann HJ. Glycine receptors influence radial migration in the embryonic mouse neocortex. Neuroreport 2011; 22: 509-513.

39. Stancik EK, Navarro-Quiroga I, Sellke R, Haydar TF. Heterogeneity in ventricular zone neural precursors contributes to neuronal fate diversity in the postnatal neocortex. J Neurosci 2010; 30: 7028-7036.

40. Gotz M, Stoykova A, Gruss P. Pax6 controls radial glia differentiation in the cerebral cortex. Neuron 1998; 21: 1031-1044.

41. Hutton SR, Pevny LH. SOX2 expression levels distinguish between neura progenitor populations of the developing dorsal telencephalon. Dev Biol 2011; 352 : 40-47.

42. Sessa A, Mao CA, Hadjantonakis AK, Klein WH, Broccoli V. Tbr2 directs conversion of radial glia into basal precursors and guides neuronal amplification by indirect neurogenesis in the developing neocortex. Neuron 2008; 60: 56-69.

43. Haubensak W, Attardo A, Denk W, Huttner WB. Neurons arise in the basal neuroepithelium of the early mammalian telencephalon: A major site of neurogenesis. Proc Natl Acad Sci USA 2004; 101: 3196-3201.

44. Noctor SC, Martinez-Cerdeno V, Ivic L, Kriegstein AR. Cortical neurons arise in symmetric and asymmetric division zones and migrate through specific phases. Nat Neurosci 2004; 7 : 136-144.

45. Schroder S, Hoch W, Becker CM, Grenningloh G, Betz H. Mapping of antigenic epitopes on the alpha 1 subunit of the inhibitory glycine receptor. Biochemistry 1991; $\mathbf{3 0}$ : $42-47$

46. Bortone D, Polleux F. KCC2 expression promotes the termination of cortical interneuron migration in a voltage-sensitive calcium-dependent manner. Neuron 2009; 62: $53-71$.

47. Liodis P, Denaxa M, Grigoriou M, Akufo-Addo C, Yanagawa Y, Pachnis V. Lhx6 activity is required for the normal migration and specification of cortical interneuron subtypes. $J$ Neurosci 2007; 27: 3078-3089.

48. Peyre $\mathrm{E}$, Morin $\mathrm{X}$. An oblique view on the role of spindle orientation in vertebrate neurogenesis. Dev Growth Differ 2012; 54: 287-305.

49. Godin JD, Colombo K, Molina-Calavita M, Keryer G, Zala D, Charrin BC et al. Huntingtin is required for mitotic spindle orientation and mammalian neurogenesis. Neuron 2010; 67: 392-406

50. Betizeau M, Cortay V, Patti D, Pfister S, Gautier E, Bellemin-Menard A et al. Precursor diversity and complexity of lineage relationships in the outer subventricular zone of the primate. Neuron 2013; 80: 442-457.

51. Harvey RJ, Yee BK. Glycine transporters as novel therapeutic targets in schizophrenia, alcohol dependence and pain. Nat Rev Drug Discov 2013; 12: 866-885.

52. Young-Pearse TL, Ivic L, Kriegstein AR, Cepko CL. Characterization of mice with targeted deletion of glycine receptor alpha 2. Mol Cell Biol 2006; 26: 5728-5734.

53. Platel JC, Boisseau S, Dupuis A, Brocard J, Poupard A, Savasta M et al. $\mathrm{Na}+$ channelmediated $\mathrm{Ca} 2+$ entry leads to glutamate secretion in mouse neocortical preplate. Proc Natl Acad Sci USA 2005; 102: 19174-19179.

54. McDearmid JR, Liao M, Drapeau P. Glycine receptors regulate interneuron differentiation during spinal network development. Proc Natl Acad Sci USA 2006; 103: 9679-9684.

55. Fang WQ, Chen WW, Fu AK, Ip NY. Axin directs the amplification and differentiation of intermediate progenitors in the developing cerebral cortex. Neuron 2013; 79: 665-679.

56. Dehay C, Kennedy H. Cell-cycle control and cortical development. Nat Rev Neurosci 2007; 8: $438-450$

57. Lukaszewicz A, Savatier P, Cortay V, Kennedy H, Dehay C. Contrasting effects of basic fibroblast growth factor and neurotrophin 3 on cell cycle kinetics of mouse cortical stem cells. J Neurosci 2002; 22: 6610-6622.

58. Lange C, Huttner WB, Calegari F. Cdk4/cyclinD1 overexpression in neural stem cells shortens G1, delays neurogenesis, and promotes the generation and expansion of basal progenitors. Cell Stem Cell 2009; 5: 320-331.

59. Pilaz LJ, Patti D, Marcy G, Ollier E, Pfister S, Douglas RJ et al. Forced G1-phase reduction alters mode of division, neuron number, and laminar phenotype in the cerebral cortex. Proc Natl Acad Sci USA 2009; 106: 21924-21929.

60. Cossart R. The maturation of cortical interneuron diversity: how multiple developmental journeys shape the emergence of proper network function. Curr Opin Neurobiol 2011; 21: 160-168

61. Sauer JF, Bartos M. Postnatal differentiation of cortical interneuron signalling. Eur $J$ Neurosci 2011; 34: 1687-1696. 
62. Seybold BA, Stanco A, Cho KK, Potter GB, Kim C, Sohal VS et al. Chronic reduction in inhibition reduces receptive field size in mouse auditory cortex. Proc Natl Acad Sci USA 2012; 109: 13829-13834.

63. Chattopadhyaya B, Cristo GD. GABAergic circuit dysfunctions in neurodevelopmental disorders. Front Psychiatr 2012; 3: 51.

64. Gogolla N, Leblanc JJ, Quast KB, Sudhof TC, Fagiolini M, Hensch TK. Common circuit defect of excitatory-inhibitory balance in mouse models of autism. J Neurodev Disord 2009; 1: $172-181$.

65. Marin O. Interneuron dysfunction in psychiatric disorders. Nat Rev Neurosci 2012; 13: 107-120.
66. Cobos I, Calcagnotto ME, Vilaythong AJ, Thwin MT, Noebels JL, Baraban SC et al. Mice lacking Dlx1 show subtype-specific loss of interneurons, reduced inhibition and epilepsy. Nat Neurosci 2005; 8: 1059-1068.

67. Del Pino I, Garcia-Frigola C, Dehorter N, Brotons-Mas JR, Alvarez-Salvado E, Martinez de Lagran $\mathrm{M}$ et al. Erbb4 deletion from fast-spiking interneurons causes schizophrenia-like phenotypes. Neuron 2013; 79: 1152-1168.

68. Rico B, Marin O. Neuregulin signaling, cortical circuitry development and schizophrenia. Curr Opin Genet Dev 2011; 21: 262-270.

69. Godin JD, Thomas N, Laguesse S, Malinouskaya L, Close P, Malaise O et al. p27(Kip1) is a microtubule-associated protein that promotes microtubule polymerization during neuron migration. Dev Cell 2012; 23: 729-744. 